\title{
Robotic Sorting on the Grid
}

\author{
Jared Coleman \\ jaredcol@usc.edu \\ Department of Computer Science, University of Southern \\ California \\ Los Angeles, California, USA
}

\author{
Oscar Morales-Ponce \\ oscar.moralesponce@csulb.edu \\ Department of Computer Science, California State \\ University \\ Long Beach, California, USA
}

\begin{abstract}
Inspired by robotic applications, we study the problem of sorting a set of items over a physical domain with mobile agents. Given $m$ mobile robots and a grid where each cell contains a single element, the objective is to design algorithms that allow robots to cooperatively sort the elements over the grid in the minimum time. We assume a synchronous model where robots do not communicate, can carry up to $c$ elements, and can move between adjacent cells in one unit of time (grab and release time is negligible). First, we show that any algorithm requires at least $\Omega\left(\frac{n^{2}}{m c}\right)$ units of time to sort an $n$-element line (an $n \times 1$ grid) and present an algorithm that sorts the elements in $O\left(\frac{n^{2}}{m c}\right)$ time. Then, we show that any $n \times n$-grid requires at least $\Omega\left(\frac{n^{3}}{m c}\right)$ time and present an algorithm that completes in $O\left(\frac{n^{3} \log n}{m c}\right)$ time. Our algorithms have an equivalent competitive ratio to Shear Sort [Isaacd et al., Proc ICPP 1986] with only $m=n$ agents (compared to the $n^{2}$ processors required by Shear Sort). Finally, we present experimental results that show the capacity has very little impact on the overall runtime and that a simplification of the algorithm leads to better results.
\end{abstract}

\section{CCS CONCEPTS}

- Theory of computation $\rightarrow$ Distributed algorithms.

\section{KEYWORDS}

Robot, Multi-Agent, Sorting, Arranging, Grid, Cooperative

\section{ACM Reference Format:}

Jared Coleman and Oscar Morales-Ponce. 2022. Robotic Sorting on the Grid. In 23rd International Conference on Distributed Computingand Networking (ICDCN 2022), January 4-7, 2022, Delhi, AA, India. ACM, New York, NY, USA, 5 pages. https://doi.org/10.1145/3491003.3491016

\section{INTRODUCTION}

Fleets of robots are currently in use in different applications. In particular, warehouses use fleets of robots to pick up customer orders [4]. Items in a warehouse are stored along corridors so that customer orders can be fulfilled by mobile robots which traverse them autonomously. Sorting items in the warehouse could considerably reduce the time and energy that robots spend traversing

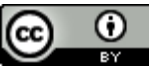

This work is licensed under a Creative Commons Attribution International 4.0 License.

ICDCN 2022, January 4-7, 2022, Delhi, AA,India

(C) 2022 Copyright held by the owner/author(s)

ACM ISBN 978-1-4503-9560-1/22/01.

https://doi.org/10.1145/3491003.3491016 corridors to fetch items. Robots may have different capabilities including carrying capacity, speed, communication, etc. Any robot with unlimited capacity could easily sort the grid by visiting each cell at most twice. The cost to maintain such a powerful robot, though, is likely unreasonable in the warehouse setting. More likely, companies would prefer to leverage a fleet of cheaper, less powerful robots that are easier to maintain. In this paper, we model the warehouse as a grid-world environment where robots can move horizontally and vertically through cells in the grid, each of which may contain an item. For simplicity of presentation, we consider a set of homogenous robots with limited carrying capacity. We consider the problem of sorting items in the minimum time possible over a physical space with a team of mobile robots. We study how carrying capacity and the number of robots impact the sorting time.

Sorting is a fundamental problem in algorithmics that under the traditional RAM model, has been generally solved for decades. In this paper, we study a variant of the sorting problem which has received considerably less attention. We consider systems of mobile robots that must cooperate to sort elements over a physical domain. Unlike the RAM model, elements cannot move to any location in the physical domain in constant time. The cost to move elements from one location to another dominates the runtime of algorithms in this domain. We consider a discrete grid-world wherein each timestep, robots can move (and carry elements) one cell at a time.

The sorting problem is foundational for many more complex problems in cooperative robotics, such as arranging, categorizing, construction, etc. Surprisingly, many of these more complex tasks have been studied, albeit with mostly empirical results for quite domain-specific applications [1-3, 5, 7-9]. We are interested in the more general sorting problem in a grid-world environment by mobile robots with limited carrying capacity. We provide both offline and online optimal algorithms for the one-dimensional world, and competitive algorithms for the two-dimensional world.

\subsection{Model}

Throughout the paper, we use $n$ to denote the size of the grid, $m$ to denote the number of homogeneous robots, and $c$ to denote the robots' capacity (the maximum number of elements a robot can carry at one time). Robots follow a look-compute-move (LCM) protocol. In each round, robots observe the environment (the elements they are carrying and the element at the cell they currently occupy), perform any computation necessary, then move (or choose not to move) to an adjacent cell in the grid. We study the sorting problem under a synchronous model where all robots perform move cycles simultaneously. The algorithms presented in this paper make no assumptions about the communication, memory, or other capabilities of robots, allowing for an elegant analysis. These results can be 
extended to handle asynchronous systems at the cost of requiring additional robot capabilities.

Initially, there exists an item in each cell in the grid. No more than one item can be placed in any cell at a time. During a robot's compute cycle, it may pick up an item from the cell it is currently occupying (if the cell is not empty and doing so would not violate the robot's capacity), place an item which it is currently carrying in the cell (if the cell is empty), or exchange one of the items it is currently carrying with the item in the cell.

\subsection{Results and Outline}

We organize the paper as follows. In Section 2 we present the related work. In Section 3, we show that any algorithm requires $\Omega\left(\frac{n^{2}}{m c}\right)$ steps to sort a line and present a $O\left(\frac{n^{2}}{m c}\right)$ running time algorithm that sorts the line. Then, in Section 4 , we show that any algorithm requires at least $\Omega\left(\frac{n^{3}}{m c}\right)$ steps to sort an $n \times n$ grid and present an $O\left(\frac{n^{3} \log n}{m c}\right)$ running time algorithm that sorts the grid.

In Section 5 we propose a simplification of the algorithm presented in Section 4, and present experimental results which suggest that the simplified algorithm is actually more efficient than the analyzed algorithm. This result suggests that, by using other techniques, tight analytical bounds for this simpler algorithm could be discovered. We leave this a topic for future work.

Finally, we conclude the paper in Section 6 with a discussion on potential avenues for future work.

\section{RELATED WORK}

Sorting is arguably the most fundamental problem in algorithmics and so has been the subject of extensive study under many different models and assumptions. Perhaps surprisingly though, the problem of sorting or arranging objects over a physical space by one or more mobile agents, has not received much attention in the literature. The most relevant work falls under two categories: parallel sorting algorithms and multi-agent path planning.

Various parallel sorting algorithms have been proposed in the literature for different architectures (mesh, cube, grid, etc.) [1]. These algorithms consider sorting by distributed but immobile computational nodes and exploit direct communication links between nodes. One of the most relevant parallel sorting algorithms to the problem studied in this paper, due to its assumption that processors are placed over a grid-like communication network, is Shear Sort, an algorithm for sorting elements over a grid of static processors which can only communicate with their neighbors [6]. The algorithm can sort an $n$-element grid in $O(n \log n)$ time, but requires $n$ immobile processors (one at each cell in the grid).

Sorting Networks [3] are parallel-sorting devices composed of individual comparison elements (which perform some comparison on a fixed number of inputs) and wires connecting these elements. Many efficient (or even optimal) sorting networks have been proposed for different problem types [2, 5, 7]. Sorting networks are capable of sorting a one-dimensional array of inputs over a network of physically distributed computational nodes, but they do not consider object arrangement over two-dimensional space.
Path planning (for one or multiple agents) is another popular area of research. [9] uses path-planning techniques to design an algorithm for sorting elements into a grid with a single robotic arm. Research in object arrangement by distributed mobile robotics systems, though, is sparse. Interesting empirical results for an insectinspired online algorithm for separating two categories of items in a grid-world environment have been reported [8]. To the best of our knowledge, though, the fundamental sorting problem by teams of mobile agents and the bounds reported in this paper have not been considered before.

\section{SORTING THE LINE SEGMENT}

In this section, we study the problem on a line segment (an $n \times 1$ grid). Recall there are $m$ robots (each with capacity $c$ ) and $n$ positions on the line segment, with each initially containing a single item to be sorted. For any initial configuration, each item is displaced from its rightful position (its position if the items were sorted on the line). Let $d(i)$ be the displacement of item $i$. This idea is helpful for studying worst-case-scenarios.

LEMmA 3.1. Every algorithm takes $\Omega\left(\frac{n^{2}}{m c}\right)$ steps to sort the items in the worst case.

Proof. Consider the initial configuration where items are unique and in reverse order. Then the total displacement is

$$
D=2 \sum_{i=1}^{\lfloor n / 2\rfloor} n+1-2 i=2\left\lfloor\frac{n}{2}\right\rfloor\left(n-\left\lfloor\frac{n}{2}\right\rfloor\right) .
$$

Observe that in a single time-step, at most $m \cdot c$ elements can move toward their final sorted destination (i.e. every robot is carrying the maximum number of items a step closer to their final destinations). Let $D_{i}$ denote the total displacement of the items after $i$ steps. Then the items are sorted once $D_{i}=0$ and $D_{i} \geq \frac{n(n-2)}{2}-i \cdot n m c$.

$$
\begin{aligned}
0 & \geq 2\left\lfloor\frac{n}{2}\right\rfloor\left(n-\left\lfloor\frac{n}{2}\right\rfloor\right)-i \cdot m c \\
i \cdot m c & \geq 2\left\lfloor\frac{n}{2}\right\rfloor\left(n-\left\lfloor\frac{n}{2}\right\rfloor\right) \\
i & \geq \frac{2}{m c}\left\lfloor\frac{n}{2}\right\rfloor\left(n-\left\lfloor\frac{n}{2}\right\rfloor\right)=\Omega\left(\frac{n^{2}}{m c}\right)
\end{aligned}
$$

We now present a solution that finishes in $O\left(\frac{n^{2}}{m c}\right)$ steps. Suppose robot $i$ starts at position $\frac{n}{m}$ and covers the range

$$
\left[\left\lfloor\frac{i(n-c)}{m}\right\rfloor,\left\lfloor\frac{(i+1)(n-c)}{m}\right\rfloor+c\right)
$$

For now, assume $n-c$ is divisible by $m$. Then each robot covers $\frac{n+c(m-1)}{m}$ positions and the entire interval is covered. Each robot independently tries to sort the section they cover and does not move beyond its bounds. Observe that robots $i$ and $i+1$ share the $c$-element interval $\left[\frac{(i+1)(n-c)}{m}, \frac{(i+1)(n-c)}{m}+c\right)$. We assume all robots move synchronously and $c<\frac{n+c(m-1)}{2 m}$, so no two robots are ever in a shared section at the same time since they maintain a constant hop-distance larger than $c$ from each other.

Consider the following algorithm 


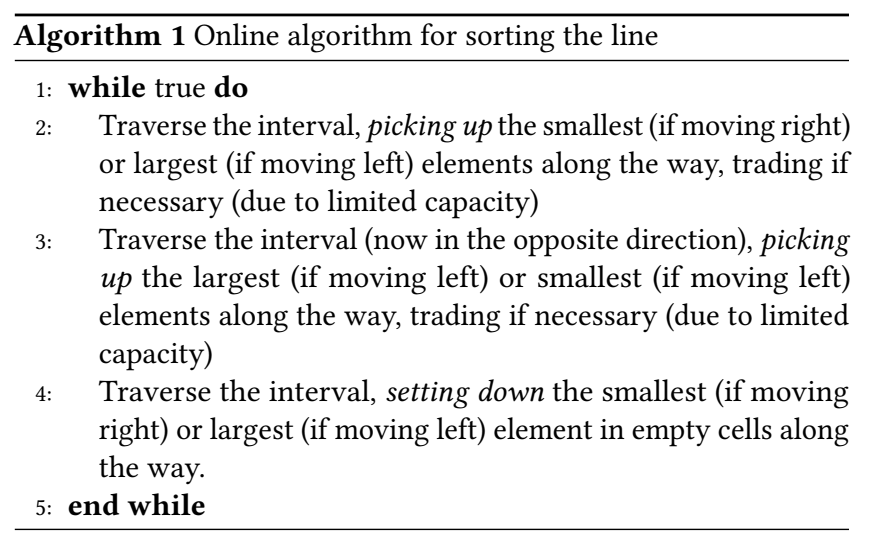

Theorem 3.2. Algorithm 1 correctly sorts the items in $\Theta\left(\frac{n^{2}}{m c}\right)$ steps.

Proof. Let a phase be $3 \frac{n+c(m-1)}{m}$ steps (the time it takes for the robot to traverse its entire section three times, as in the algorithm). In a single phase, each robot carries the greatest $c$ elements in its section into the $c$ rightmost positions of its section (and vice-versa for its smallest elements). Note that at the end of each phase, each robot is also carrying no items since by Line 4 they set down all their elements before reaching the end of the interval. Observe then, that after at most $m$ phases, the greatest and least $c$ elements are in their final positions. Further, in each subsequent phase, the next $c$ greatest and least elements are placed in their final positions. Therefore after an initial $m$ phases, $2 c$ elements are sorted in each phase and the entire segment is sorted after at most $m+\frac{n}{2 c}$ phases, or

$$
\begin{aligned}
\left(m+\frac{n}{2 c}\right) \cdot 3 \frac{n+c(m-1)}{m} & =\frac{2 c m+n}{2 c} \cdot 3 \frac{n+c m-c}{m} \\
& \leq \frac{2 c n+n}{2 c} \cdot 3 \frac{n+c n-c}{m} \\
& =O\left(\frac{n^{2}}{m c}\right)
\end{aligned}
$$

steps. Then, with Lemma 3.1, Algorithm sorts the grid in $\Theta\left(\frac{n^{2}}{m c}\right)$ steps.

To address the case where $n-c$ is not divisible by $m$, simply allow the rightmost robot to cover its assigned section and the rest of the elements until the end of the line-segment. Thus, the last robot is responsible for covering at most $2 \frac{n+c(m-1)}{m}$ elements (which is asymptotically the same). In order to maintain the property that two robots are ever both in their shared sections, robots must be synchronized so that they all start new phases at the same time. This can be achieved by forcing all other robots to wait while the final robot finishes sorting its larger section.

\section{SORTING THE GRID}

In this section, we study the problem on the grid and start by showing a lower bound:

LEMMA 4.1. Every algorithm takes $\Omega\left(\frac{n^{3}}{m c}\right)$ steps to sort the items in the worst case.

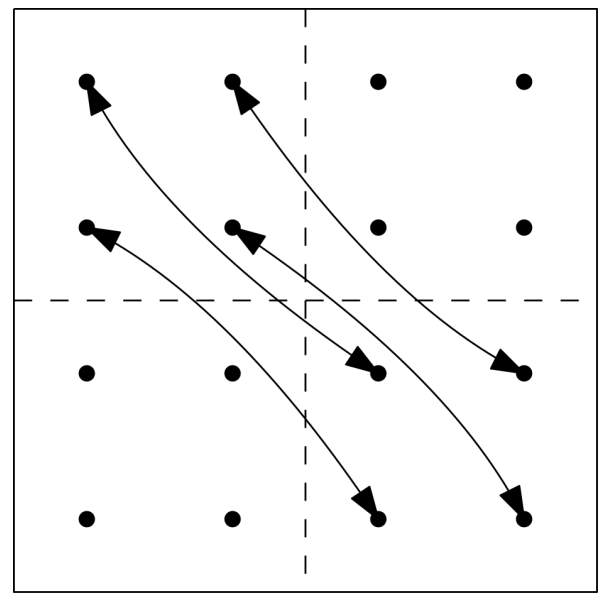

Figure 1: Construction for lower bound. Given the sorted grid of unique elements, swap quadrants diagonally so that each element is a distance $\frac{n}{2}$ away from its destination.

Proof. Consider the initial configuration where each element with destination $(i, j)$, is placed at position

$$
\left(i+\left\lfloor\frac{n}{2}\right\rfloor \bmod n, j+\left\lfloor\frac{n}{2}\right\rfloor \bmod n\right)
$$

(Figure 1). Observe each element, then, is displaced at least $n-1$ units from its destination and the total displacement of the initial configuration is $D \geq(n-1)^{3}$. Observe that in a single time-step, at most $m \cdot c$ elements can move toward their final sorted destination (i.e. every robot is carrying the maximum number of items a step closer to their final destination). Let $D_{i}$ denote the total displacement of the items after $i$ steps. Then the items are sorted once $D_{i}=0$ and $D_{i} \geq D-i \cdot n m c$.

$$
\begin{aligned}
0 & \geq D-i \cdot n m c \geq(n-1)^{3}-i \cdot m c \\
i \cdot m c & \geq(n-1)^{3} \\
i & \geq \frac{(n-1)^{3}}{m c}=\Omega\left(\frac{n^{3}}{m c}\right)
\end{aligned}
$$

Shear Sort, first introduced in 1986 is a well-known parallelized grid-sort algorithm that runs in $O(n \log n)$ time [6]. Shear Sort requires that each node in the grid contain a processor that is capable of communicating with processors in adjacent cells. The algorithm works by alternating between sorting rows and columns until the entire grid is sorted. Under the traditional model, rows and columns can be independently sorted in linear (with respect to the size of the rows/columns, $O(n)$ time. It has been shown that at most $O(\log n)$ row- and column-phases are necessary to sort the entire grid, resulting in a total of $O(n \log n)$ steps.

Shear Sort requires a processor at each cell in the grid which, in each round, may swap its value with that of an adjacent processor. Thus $m=n^{2}, c=1$, and the competitive ratio of Shear Sort is:

$$
C R_{\text {shear }} \leq \frac{n \log n}{n^{3} /(m c)}=\log n
$$



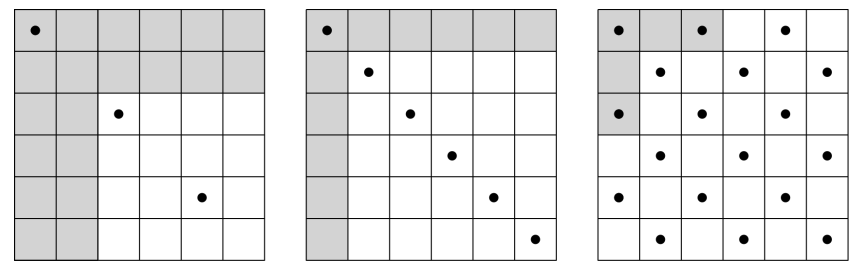

Figure 2: Example prescriptions for $m<n$ (left), $m=n$ (middle), and $m>n$ (right). The shaded areas indicate prescribed row and column sections for the top-left robot. In the rightmost example, the shaded section is given for $c=1$.

We introduce an algorithm inspired by Shear Sort that works for $m \leq n$ robots. Similar to Algorithm 1, we prescribe sections for each robot to sort.

First, we evenly space robots across the diagonal of the grid. If $m \geq n$ then a robot will be placed on each cell in the diagonal. Then, divide remaining robots evenly into $n$ groups (one for each row) and place robots in their assigned row so they are as evenly spaced across the row as possible (Figure 2). This results in robots being placed along diagonals of the grid. Then we assign row and column sections to each robot so that there is a $c$-cell overlap between its section and its neighboring robot's sections. Thus, each robot is prescribed a section of at most $\frac{n^{2}}{m}+2 c$ elements. If $m \leq n$, then each robot is responsible for sorting at least one row and column each. Observe robots do not need IDs since each simply executes the same algorithm in a different prescribed section of the grid.

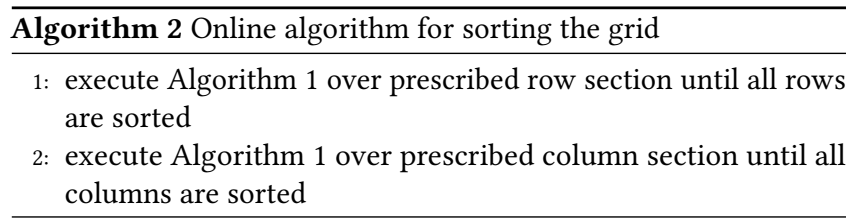

The algorithm performs best when each column and row is being sorted by at least one robot.

Lemмa 4.2. If $m \geq n$, then Algorithm 2 terminates in $O\left(\frac{n^{3} \log n}{m c}\right)$ steps.

Proof. If $m \geq n$, then each row and column has at least $\left\lfloor\frac{m}{n}\right\rfloor$ robots and by Theorem 3.2, the number of rounds to sort all rows/ columns independently (via Algorithm 1) is $O\left(\frac{n^{3}}{m c}\right)$. Therefore, the number of steps required to sort the entire grid is $O\left(\frac{n^{3} \log n}{m c}\right)$.

For smaller systems of robots, each robot may be required to sort more than one row/column in each phase. Not surprisingly, this results in a larger asymptotic runtime.

Lemмa 4.3. If $m<n$, then Algorithm 2 terminates in $O\left(\frac{n^{4} \log n}{m^{2} c}\right)$ steps.

Proof. If $m \leq n$, then a robot in the system may responsible for sorting up to $\left\lceil\frac{n^{2}}{m}\right\rceil$ rows/columns and, again by Theorem 3.2, the time to sort the grid is $O\left(\frac{n^{4} \log n}{m^{2} c}\right)$.
Then, we leverage these lemmas to make the following claim about the competitiveness of our online sorting algorithm.

THEOREM 4.4. The competitive ratio of Algorithm 2 is

$$
C R_{\text {grid }} \leq \begin{cases}\log n & \text { if } m \geq n \\ \frac{n \log n}{m^{2}} & \text { otherwise }\end{cases}
$$

Proof. Recall that Lemma 3.1 gives us a lower bound of $\frac{n^{3}}{m c}$. Then the proof follows directly from Lemmas $4.2 \& 4.3$.

REMARK. For any $m \geq n$ our algorithm is equally as competitive as Shear Sort, despite using far fewer processors (robots).

\section{IMPLEMENTATION}

Algorithm 2, while relatively simple to explain and reason about, is non-trivial to implement. Depending on the number of robots in the system, the sections covered by robots may vary in size and so the time required to sort rows columns may also vary. Even for systems with exactly $n$ robots, the exact number of rounds required to sort all rows (columns) is variable, and depends on the initial ordering of elements in the row (column). Recall, robots must switch between row-sorting and column-sorting simultaneously in order to avoid collision. In order to address this, we can add a checking routine and communication between the robots, but this adds assumptions about the capabilities of robots. Alternatively, we can configure robots to perform the sorting algorithm for a fixed number of rounds - the upper bound for sorting a section, as described in the previous section. It's likely, though, that the rows will be sorted in much fewer rounds than the required upper bound and robots will spend a lot of time doing useless work.

Interestingly, robots do not need to fully sort the entire row or column in each phase. In fact, we claim that as long as robots perform at least one sweep of the line-segment sorting algorithm in each phase, the grid will eventually be sorted.

\begin{tabular}{l}
\hline Algorithm 3 Modified online algorithm for sorting the grid \\
\hline 1: execute Algorithm 1 over prescribed row for $k$ phases \\
2: execute Algorithm 1 over prescribed column for $k$ phases \\
\hline
\end{tabular}

LEMmA 5.1. Algorithm 3 sorts the grid in a finite number of phases.

Proof. Observe that in each phase, the largest and smallest unsorted items move toward their destinations. Inductively, it is simple to verify that the grid eventually becomes sorted.

We implemented Algorithm 3 for systems with $n$ robots (so that they can be placed along the diagonal of the grid and there is exactly one robot per row and column). We run the algorithm on grids with widths in $\{1,2, \ldots, 10\}$, capacity $\{1,2, \ldots, n\}$, and $k \in\left\{1, \frac{1}{10} n, \frac{2}{10} n, \ldots, n\right\}$ (where $n$ is the number of elements in the grid, width $\times$ width). For each grid size, we run the algorithm 50 times with different random initial configurations (uniformly random permutation of all possible element location assignments). The results are summarized in Figure 3. Interestingly, the algorithm performs best for the simplest implementation, $k=1$. Further, as the size of the grid increases, the value of $k$ has an even greater effect on the sorting time of the algorithm. 


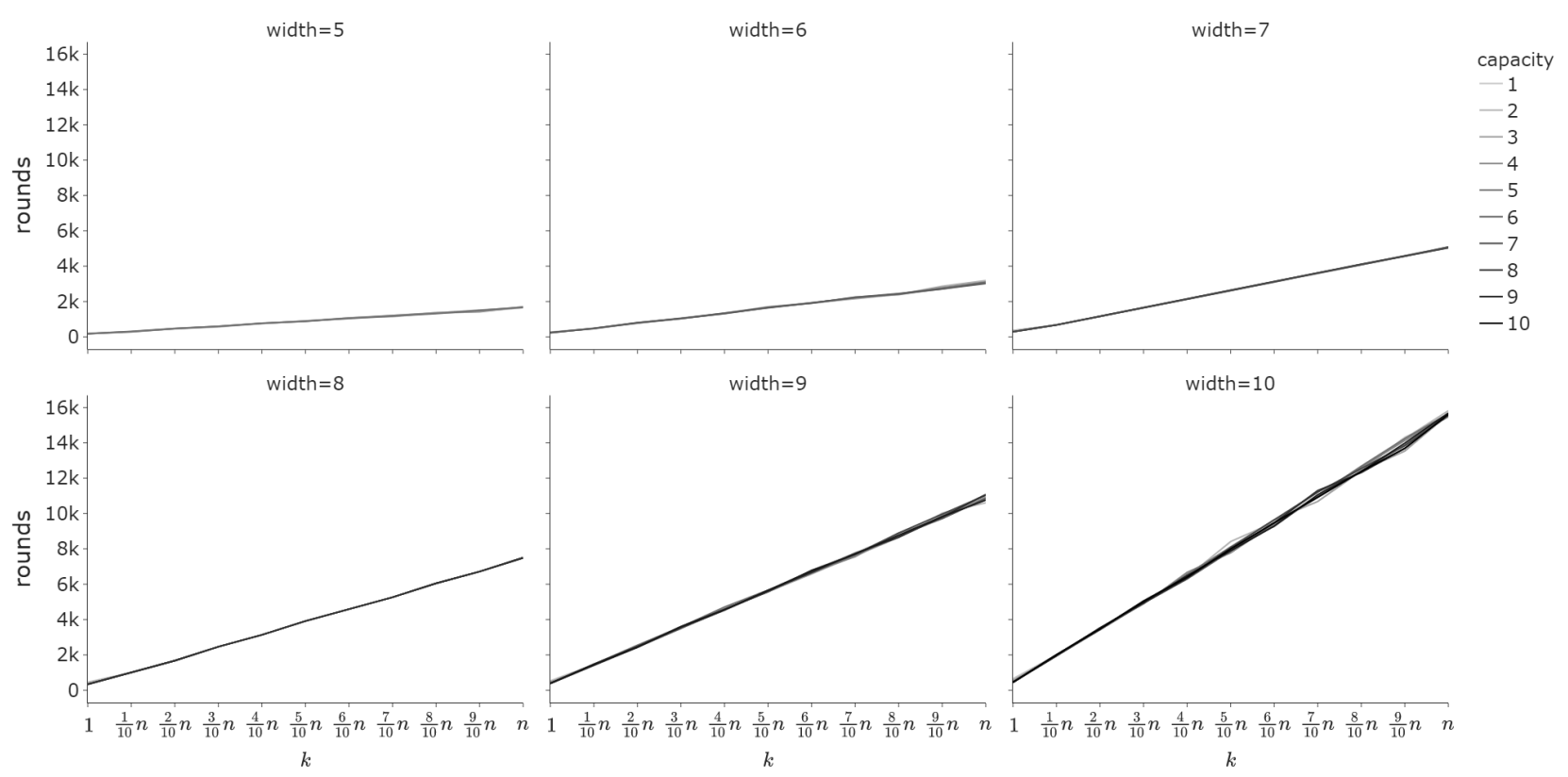

Figure 3: Algorithm performs best when $k=1$

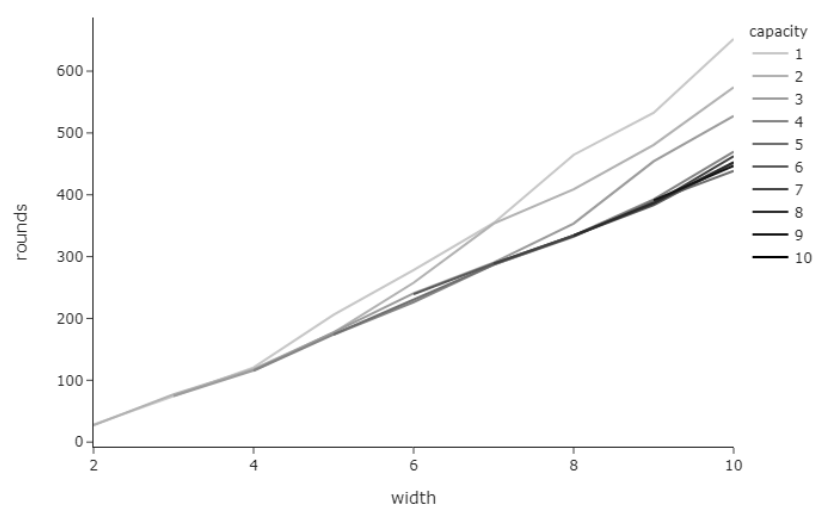

Figure 4: Plot which shows how capacity affects the number of rounds required to sort grids with varying widths $(k=1)$. It appears that as the size of the grid increases, so too does the impact of capacity on sorting time.

As expected, a higher robot capacity results in fewer rounds to sort on average (Figure 4).

Interestingly, though, the effect of capacity is dwarfed by the that of value of $k$. In the experiments, the capacity affects the number of rounds required to sort by at most 200 rounds (capacity 6 vs 1 on a $10 \times 10$ grid). The $k$-value, however, affects the runtime of the algorithm by more than 14 thousand rounds $\left(k=1\right.$ vs $k=n^{2}$ on a $10 \times 10$ grid)!

\section{CONCLUSION}

In this paper, we provide a theoretical basis for the fundamental problem of collaboratively sorting elements over a physical space with a system of mobile robots. There are a few avenues for future research in this area. Sorting is a fundamental task for many other more complex tasks. Further study is required to understand the consequences of these results for problems like collaborative arrangement, construction, categorizing, etc. Finally, we consider only discrete grid-world environments in this paper. It would be interesting to consider the sorting problem (and its extensions) in continuous domains.

\section{REFERENCES}

[1] Selim G Akl. 2014. Parallel sorting algorithms. Vol. 12. Academic press.

[2] Sherenaz W Al-Haj Baddar and Kenneth E Batcher. 2012. Designing sorting networks: A new paradigm. Springer Science \& Business Media.

[3] Kenneth E Batcher. 1968. Sorting networks and their applications. In Proceedings of the April 30-May 2, 1968, spring joint computer conference. 307-314.

[4] Robert Bogue. 2016. Growth in e-commerce boosts innovation in the warehouse robot market. Industrial Robot: An International fournal (2016).

[5] Daniel Bundala and Jakub Závodnỳ. 2014. Optimal sorting networks. In International Conference on Language and Automata Theory and Applications. Springer, 236-247.

[6] Scherson Isaacd, Sen Sandeep, and AD Shamir. 1986. Shear sort-a true twodimensional sorting technique for VLSI networks. In International Conference on Parallel Processing. International Conference on Parallel Processing, 903-908.

[7] Rene Mueller, Jens Teubner, and Gustavo Alonso. 2012. Sorting networks on FPGAs. The VLDB fournal 21, 1 (2012), 1-23.

[8] Tao Wang and Hong Zhang. 2004. Collective sorting with multiple robots. In 2004 IEEE International Conference on Robotics and Biomimetics. IEEE, 716-720.

[9] Haojian Zhang, Tingting Su, Shaohong Wu, Jun Zheng, and Yunkuan Wang. 2018. Simultaneous path planning and trajectory optimization for high-speed sorting system. International fournal of Advanced Robotic Systems 15, 5 (2018), 1729881418797870. 